\title{
Review
}

\section{Gene therapy of hepatic diseases: prospects for the new millennium}

As we stand on the cusp of the 21 st century, the prospect of treating liver diseases by the manipulation of genetic material seems to be not merely a remote possibility, but a tangible reality. The rapid advances in biotechnology over the past few decades have afforded a unique understanding of the molecular mechanisms underlying various hepatic disorders. As a result, novel and exciting techniques have been developed for the genetic modification of hepatocytes. In this article, we will highlight advances in particular areas, and lend perspective as to their future prospects.

\section{Strategies for hepatic gene therapy}

GENE AUGMENTATION THERAPY

This strategy involves administration of a normal gene to replace a missing or dysfunctional gene product resulting from a defective gene, as has been illustrated by studies on the hereditary disorder familial hypercholesterolaemia. In this disease, a defect in the low density lipoprotein (LDL) receptor gene results in abnormal expression of the LDL receptor and consequent failure of clearance of LDL cholesterol. ${ }^{1}$ Using an animal model of familial hypercholesterolaemia, the Watanabe heritable hyperlipidaemic (WHHL) rabbit, investigators have been able to show the successful transduction of a functional rabbit LDL gene into target hepatocytes. This resulted in a $30-40 \%$ reduction in serum cholesterol, with the recombinant LDL receptor being detectable for up to six months. ${ }^{2}$ In clinical trials, five patients homozygous for familial hypercholesterolaemia underwent ex vivo replacement of the faulty gene. ${ }^{3}$ This was achieved by segmental hepatic resection, preparation of hepatocyte cultures, and transduction of these cultures with a recombinant retrovirus encoding the gene for the human LDL receptor. The genetically modified cells were then transplanted into the liver using portal venous cannulation. Prolonged reductions in LDL cholesterol were seen in three of the five patients, and this procedure was remarkably free of any major side effects. This study served to demonstrate the feasibility of ex vivo therapy, although concerns remain regarding the long term efficacy of this approach. Another disadvantage is the limited availability of autologous hepatocytes. However, recent advances in the propagation of liver cells such as the introduction of temperature sensitive mutant Simian virus 40 (SV-40) $\mathrm{T}$ antigen mutants ${ }^{4}$ may obviate the need for harvests of large numbers of host cells for genetic manipulation.

In vivo approaches to genetic manipulation involve the transfer of genes to target tissue by either systemic administration or direct injection. The paradigm for in vivo therapy has been the approach to ornithine transcarbamylase (OTC) deficiency. Using the sparse-fur mouse model of OTC deficiency, an intravenous injection of an adenoviral vector has been shown to produce a high level gene transfer, leading to expression of the functional enzyme. ${ }^{5}$

\footnotetext{
^Note added in proof: A troubling development has occurred in a clinical trial of adenoviral gene therapy for ornithine transcarbamylase deficiency. The unexplained death of one of the trial patients has resulted in the indefinite suspension of the trial.
}

Based on these experiments, a human clinical trial has recently been approved for the genetic treatment of adults with partial OTC deficiency. When available, the results of this study will provide further valuable insights into the clinical applications of this technology. *

Another recent in vivo experiment demonstrated the use of retroviral vectors to produce sustained expression of therapeutic levels of factor VIII in a neonatal mouse model of haemophilia A. ${ }^{6}$ As it is desirable to institute gene therapy early in life, the propagation of neonatal hepatocytes represents a promising approach with clinical relevance.

REPAIR OF ABNORMAL GENES

An ideal approach to gene therapy would be to repair the defective gene. This is particularly true for dominant negative mutations where simple introduction of a normal gene product may not overcome the dysfunction caused by the continued production of an abnormal product. Such repair processes do occur naturally, but at very low frequency. Recently, a novel technique has been devised to correct a faulty gene by harnessing the repair mechanisms of the host. In this approach, a molecule is prepared composed of both DNA and RNA domains forming a chimeric RNA/DNA oligonucleotide. ${ }^{7}$ The RNA domain of the oligonucleotide is designed to be perfectly complementary to the targeted gene except for a single base mismatch. The mismatch seems to trigger the cell's DNA repair mechanism, and induces a nucleotide substitution at the target site. In an in vitro application of this technique, chimeric RNA/DNA oligonucleotides were used to introduce successfully a point mutation into the chromosomal alkaline phosphatase gene in a human hepatoma cell line. ${ }^{8}$ The base conversion efficiency was calculated to be approximately $40 \%$ based on amplification and cloning. This approach was extended to an in vivo setting, targeted against the rat factor IX gene. ${ }^{9}$ The desired mutation was noted in up to $40 \%$ of target sites. However, a similar degree of success has not been duplicated outside of that laboratory. It is possible that the methods used to assess efficiency overestimate the actual figure. Nevertheless, the studies represent an important landmark in the field of genetic research.

INHIBITION OF ENDOGENOUS GENE EXPRESSION

Acquired liver disorders represent a more heterogeneous array of conditions, some of which may be amenable to genetic therapy by inhibiting the expression of various genes. Examples include infections with the hepatitis viruses where viral gene products can be blocked, and malignancies in which an oncogene or other growth regulatory genes can be inhibited. Several types of molecular agents have been developed.

Abbreviations used in this review: $\mathrm{AAV}$, adeno-associated virus; AsGPr, asialoglycoprotein receptor; BUGT, bilirubin-UDPglucuronyltransferase; GSD, glycogen storage disease; $\mathrm{HCV}$, hepatitis $\mathrm{C}$ virus; HSV-tk, herpes simplex virus thymidine kinase; hGAA, human acid $\alpha$-glucosidase; IL, interleukin; LDL, low density lipoprotein; OTC, ornithine transcarbamylase; SV-40, Simian virus 40. 
Ribozymes

Ribozymes are single stranded RNA molecules that contain a cleavage domain flanked by target binding domains on each side. These molecules can destroy target messenger RNA sequences in a catalytic manner. However, their use is limited by the susceptibility of RNA to nuclease degradation. Recently, ribozyme analogues composed of DNA (DNA ribonucleases) which are less sensitive to degradation have been developed. ${ }^{10}$ The latter have been demonstrated in vitro to inhibit hepatitis B viral gene expression substantially. ${ }^{11}$

\section{Antisense oligonucleotides}

Antisense oligonucleotides have also been successfully used to block endogenous gene expression in the Peking duck and woodchuck animal models of chronic hepatitis B. ${ }^{12}{ }^{13}$ This involves preparing a short single stranded DNA segment, or a gene which will generate RNA, which is complementary to a target messenger RNA. Within the cell, the antisense nucleic acid hybridises with and specifically inhibits translation of the particular gene product. There have been reports of systemic infusion of antisense oligonucleotides resulting in substantial liver uptake. ${ }^{14}$ In addition, targeted delivery of inhibitory oligonucleotides has been demonstrated in vitro raising the possibility of decreasing potential side effects at other non-hepatic sites. Such targeted antisense nucleotides directed against the 5'-non-translated region of hepatitis $\mathrm{C}$ virus (HCV) were shown to inhibit $\mathrm{HCV}$ protein synthesis effectively. ${ }^{15}$ Targeted inhibition of hepatitis B viral gene expression has also been demonstrated in vitro and in vivo. However, the low efficiency and possible immune response to the carriers are potential drawbacks.

VIRAL ENZYME PRODRUG THERAPY

A gene therapy strategy for the treatment of primary and metastatic liver cancer has been developed in which tumour cells are transduced with a non-mammalian "suicide" gene which can convert a non-toxic prodrug into a chemotherapeutic agent exclusively within the target malignant cells. ${ }^{16}$ As a result, the systemic toxicity often associated with chemotherapy may be avoided. For example, the herpes simplex virus thymidine kinase (HSV-tk) gene which phosphorylates the synthetic guanine analogue, ganciclovir, into a toxic metabolite ${ }^{17}$ and cytosine deaminase which converts 5-fluorocytosine into the chemotherapeutic agent 5-fluorouracil ${ }^{16}$ have been adapted for this purpose. The genes for these enzymes when introduced into malignant cells have been shown to generate sufficient toxin to destroy those cells selectively. In addition, a bystander effect has been shown to occur in which surrounding tumour cells are also affected. In this way, even cells not transfected with the gene can be destroyed. Nevertheless, the prospect of eliminating every malignant cell will depend on high efficiency of transfection.

Co-delivery of genes encoding antitumour cytokines (e.g. interleukin-2 (IL-2) and granulocyte-macrophage colony stimulating factor) in an attempt to stimulate antitumour immunity ${ }^{18}$ has also been developed. One recent study using this so-called "cancer vaccine" approach used a murine model transfected by both HSV-tk and IL-2. Major tumour regression was noted and was associated with the apparent development of systemic antitumoral immunity. Further studies will be required to determine whether this approach will have clinical applications.

\section{Methods of gene delivery to the liver}

The importance to successful gene therapy of developing effective gene delivery systems cannot be overstated. An ideal vector would be able to deliver genetic material efficiently and specifically, and would result in high level, properly regulated and prolonged expression. This vector should be non-toxic, non-immunogenic, and have a broad host range.

VIRAL VECTORS

Viruses have many advantages as vehicles for the delivery of genes, as this is their normal function. There have been a number of recent advances in this area.

\section{Retroviral vectors}

The ability to integrate into the host genome ${ }^{19}$ and to be transmitted to progeny cells has made retroviral vectors very popular. Their obvious advantages of efficient and specific gene delivery are, however, offset by certain drawbacks. The most important of these is that retroviruses are unable to infect non-dividing cells. This has led to the administration of substances to stimulate hepatocyte proliferation $^{20}$ and the development of lentiviral vectors. ${ }^{21}$ Lentiviruses are a class of complex retroviruses, the best known of which is HIV-1. They are able to infect non-dividing terminally differentiated tissue such as the liver while retaining the other advantages of retroviral vectors. Lentiviruses seem to have an excellent biosafety profile. However, as there is no animal model for HIV-1 infection, the efficacy and safety of these vectors can only be tested in clinical trials. Another potential drawback associated with both retroviral and lentiviral vectors is their random integration into the recipient genome. This has led to concerns that this integration may activate oncogenes or inactivate tumour suppressor genes.

\section{Adenoviral vectors}

Adenoviruses 2 and 5 (subgroup C) are commonly used as gene vectors. Recombinant adenoviruses may be generated in high titres, and these viruses localise predominantly in the liver after systemic administration. ${ }^{22}$ The transfer of genetic material is usually quick and efficient. Despite these obvious advantages, the use of adenoviral vectors has been limited by several factors. Owing to the episomal nature of the virus, transgene expression is transient. ${ }^{23}$ The prototype viruses were immunogenic, resulting in a strong host response. ${ }^{24}$ Several strategies to attenuate the host's immune response have been studied. These include the use of transient immunosuppression by agents such as tacrolimus (FK 506) ${ }^{25}$ and the modification of the virus by deleting certain genes. ${ }^{26}$ This so-called "gutless" adenovirus is, however, still able to evoke an antiviral immune response. Another strategy is the enhancement of immunomodulatory genes produced by the adenovirus itself. ${ }^{27}$ If these immunomodulatory genes are enhanced, allogeneic cell rejection has been shown to be greatly reduced. Another important recent study has focused on the treatment of glycogen storage disease II (GSD-II) using a modified adenovirus vector encoding human acid $\alpha$-glucosidase (hGAA). ${ }^{28}$ In a knockout mouse model of GSD II, hepatic transduction with the adenoviral vector resulted in high level secretion of the precursor form of the hGAA enzyme into the circulation, with consequent peripheral uptake by skeletal muscle and decreased glycogen accumulation in affected muscles. This approach is diametrically different to previous studies in this area, in that it has targeted the liver, rather than affected fibroblasts. If confirmed by other researchers, these findings offer exciting therapeutic possibilities for these otherwise incurable metabolic disorders.

Adeno-associated viral vectors

Adeno-associated virus (AAV) is a human non-pathogenic replication-defective parvovirus. One of the major potential 
advantages of this system is the property of site specific integration in the presence of a rep gene product. The wild type viral DNA can integrate preferentially into human chromosome 19. However, recombinant AAV integrates randomly when the rep gene is absent. Other advantages of recombinant AAV vector system are that the virus is nonpathogenic, does not require dividing cells, has a broad host range, and is capable of infecting many cell types. Wang and colleagues ${ }^{29}$ showed that a single intraportal injection of a recombinant AAV vector encoding canine factor IX complementary DNA under the control of a liver specific enhancer/promoter led to the long term and complete correction of haemophilia in a mouse model. Up to $15-20 \mathrm{mcg} / \mathrm{ml}$ of canine factor IX was detected in the plasma of mice for more than five months after intravenous injection of an AAV vector. The activated partial thromboplastin time of treated mice was also corrected.

\section{Simian virus 40 vectors}

This vector system utilises a replication deficient variant of SV $-40^{30}$ which contains a powerful endogenous promoter. Mice inoculated with this vector showed a high $(>90 \%)$ transduction efficiency. For example, recombinant SV-40 expressing human bilirubin-UDP-glucuronyltransferase (SV-hBUGT) was delivered to Gunn rats, which are deficient in BUGT, and hence have hyperbilirubinaemia. After sequential inoculations, great and prolonged decreases in bilirubin concentrations were noted, demonstrating the efficacy of transduction. ${ }^{30}$

The advantages of this vector are that it may be produced in high titre, and can be used against a large variety of cells. Its major drawback is its small genome, which limits insert size.

\section{Hybrid viruses}

Recently, a novel chimeric adeno-retroviral vector system, ${ }^{31}$ a chimeric adenovirus/AAV hybrid vector, ${ }^{32}$ and a HSV-1/ AAV hybrid ${ }^{33}$ have been developed. These hybrid vectors aim to combine the advantages of the component systems, while nullifying the potential drawbacks. For example, the recently developed adenovirus/AAV hybrid ${ }^{32}$ has a higher transduction efficiency and greater cloning capacity than either virus alone. In the future, these systems should prove to be useful vehicles for gene therapy.

\section{NON-VIRAL METHODS OF GENE DELIVERY}

To overcome the various problems inherent to viral delivery systems, investigators have explored artificial, non-viral gene delivery systems. The most common technique involves the attachment of a therapeutic gene to a carrier. These carriers may be either polymer based cationic carriers (conjugates) or lipid based vectors (liposomes). ${ }^{34}$

In order to improve the specificity of these vectors, the process of receptor mediated endocytosis has been studied $^{35}$ using the asialoglycoprotein receptor (AsGPr) which is expressed selectively on hepatocytes. Specific ligands recognised by the AsGPr have been attached to therapeutic genes, usually in combination with a polycation such as polylysine. The DNA-polylysine-ligand complex binds to the receptor, and is taken up by endocytosis. A fraction of the DNA diffuses into the nucleus where it is then expressed. Antisense oligonucleotides may also be targeted specifically to the liver via the AsGPr. Administration of the antisense oligonucleotide blocked the expression of hepatitis B virus in vitro, which consequently reduced the viral burden. ${ }^{11}$ Addition of endosomolytic and biocompatible solubilising agents have improved efficiency of expression. None the less, relatively low levels of expression remain a drawback of the system in vivo.

\section{Conclusions}

As with all biomedical endeavours, the leap from bench to bedside is a giant one. The last decade of this millennium has seen the science of hepatic genetics come of age. It is true that many challenges remain, but if the past is any indication, they will be countered by the persistence and ingenuity of the investigators in this field. Based on the many advances in the field, there is reason for continued, but guarded optimism for clinical applications of gene therapy for hepatic diseases.

\section{K SHETTY} G Y WU C H WU

Department of Medicine,

Division of Gastroenterology-Hepatology,

University of Connecticut Health Center,

263 Farmington Avenue

Farmington, CT 06030, USA

Correspondence to: Dr Catherine Wu. email: cwu@nso1.uchc.edu

1 Hobbs HH, Brown MS, Goldstein JL. Molecular genetics of the LDL receptor gene in familial hypercholesterolemia. Hum Mutat 1992;1:445-66. 2 Wilson JM, Roy Chowdhury N, Grossman M, et al. Temporary amelioration of hyperlipidemia in low density lipoprotein receptor deficient rabbits transplanted with genetically modified hepatocytes. Proc Natl Acad Sci USA 1990;87:8437-41

3 Grossman M, Rader DJ, Muller DW, et al. A pilot study of ex vivo gene therapy for homozygous familial hypercholestrolemia. Nat Med 1995;1: $1148-54$.

4 Tada K, Roy-Chowdhury N, Prasad V, et al. Long-term amelioration of bilirubin glucuronidation defect in Gunn rats by transplanting genetically modified immortalized autologous hepatocytes. Cell Transplant 1998;7: 607-16.

5 Raper SE, Wilson JM, Yudkoff M, et al. Developing adenoviral-mediated in vivo gene therapy for ornithine transcarbamylase deficiency. F Inherit Metab Dis 1998;21(supp 1):119-37.

6 VandenDriessche T, Vanlembrouck V, Goovaerts I, et al. Long-term expression of human coagulation factor VIII and correction of hemophilia A after in vivo retroviral gene transfer in factor-VIII deficient mice. Proc Natl Acad in vivo retroviral gene transfer

7 Yoon K, Cole-Strauss A, Kmiec EB. Targeted gene correction of episomal DNA in mammalian cells mediated by a chimeric RNA/DNA oligonucleotide. Proc Natl Acad Sci USA 1996;93:2071-6.

8 Kren BT, Cole-Strauss A, Kmiec EB, et al. Targeted nucleotide exchange in the alkaline phosphatase gene of $\mathrm{HuH}-7$ cells mediated by a chimeric RNA/DNA oligonucleotide. Hepatology 1997;25:1462-8

9 Kren BT, Bandyopadhyay P, Steer CJ. In vivo site-directed mutagenesis of the factor IX gene by chimeric RNA/DNA oligonucleotides. Nat Med 1998;4:285-90.

10 Santoro SW, Joyce GF. A general purpose RNA-cleaving DNA enzyme. Proc Natl Acad Sci USA 1997;94:4262-6.

11 Asahina Y, Ito $\mathrm{Y}$, Wu $\mathrm{CH}$, et al. DNA ribonucleases that are active against intracellular hepatitis B viral RNA targets. Hepatology 1998;28:547-54.

$12 \mathrm{Wu}$ GY, Wu CH. Specific inhibition of hepatitis B viral gene expression in vitro by targeted antisense oligonucleotides. $\mathcal{F}$ Biol Chem 1992;267:12436-9.

13 Offensperger WB, Offensperger S, Walter E, et al. In vivo inhibition of duck hepatitis B virus replication and gene expression by phosphorothioate hepatitis B virus replication and gene expression by phosphorothioat
modified antisense oligodeoxynucleotides. EMBO $₹$ 1993;12:1257-62.

14 Zhang H, Hanecak R, Brown-Driver V, et al. Antisense oligonucleotide inhibition of hepatitis $\mathrm{C}$ virus (HCV) gene expression in livers of mice infected with an HCV-vaccinia virus recombinant. Antimicrob Agents Chemother 1999;43:347-53.

$15 \mathrm{Wu}$ CH, Wu GY. Targeted inhibition of hepatitis C virus-directed gene expression in human hepatoma cell lines. Gastroenterology 1998;114:130412 .

16 Huber BE, Richards CA, Austin EA. Virus directed enzyme/prodrugtherapy (VDEPT). Selectively engineering drug sensitivity into tumors. Ann NY Acad Sci 1994;716:104-14.

17 Kaneko S, Hallenbeck P, Kotani T, et al. Adenovirus-mediated gene therapy of hepatocellular carcinoma using cancer-specific gene expression. Cancer Res 1995;55:5283-7.

18 Hurford RK Jr, Dranoff G, Mulligan RC, et al. Gene therapy of metastatic cancer by in vivo retroviral gene targeting. Nat Genet 1995;10:430-5.

19 Alpana GV. Retroviral vectors for liver-directed gene therapy. Semin Liver Dis 1999;19:27-37.

20 Patijn GA, Lieber A, Schowalter DB, et al. Hepatocyte growth factor induces hepatocyte proliferation in vivo and allows for efficient retroviral-mediated gene transfer in mice. Hepatology 1998;28:707-16.

21 Verma IM, Somia N. Gene therapy-promises, problems and prospects. Nature 1997;389:239-42.

22 Herz J, Gerard RD. Adenovirus-mediated transfer of low density lipoprotein receptor gene acutely accelerates cholestrol clearance in normal mice. Proc Natl Acad Sci USA 1993;90:2812-16.

23 Jaffe HA, Danel C, Longenecker G, et al. Adenovirus-mediated in vivo gene transfer and expression in normal rat liver. Nat Genet 1992;1:372-8.

24 Yang Y, Li Q, Ertl HCJ, et al. Cellular and humoral immune responses to viral antigen create barriers to lung-directed gene therapy with recombinant adenoviruses. $\mathcal{f}$ Virol 1995;67:2004-15.

25 Ilan Y, Jona VK, Sengupta K, et al. Transient immunosuppression with FK506 permits long-term expression of therapeutic genes introduced into the liver using recombinant adenoviruses. Hepatology 1997;26:949-56.

26 Yang Y, Nunes FA, Berencsi K, et al. Inactivation of E2a in recombinant adenoviruses limits cellular immunity and improves the prospect of gene therapy of cystic fibrosis. Nat Genet 1994;7:362-9. 
27 Lee MG, Abina MA, Haddada $\mathrm{H}$, et al. The constitutive expression of the immunomodulatory gp $19 \mathrm{~K}$ protein in E1-, E3-adenoviral vectors strongly reduces the host cytotoxic T cell response against the vector. Gene Ther

1995;2:256-62.

Amalfitano A, McVie-Wylie AJ, Hu H, et al. Systemic correction of the muscle disorder glycogen storage disease type II after hepatic targeting of a modified adenovirus vector encoding human acid-alpha-glucosidase. Proc Natl Acad Sci USA 1999;96:8861-6.

29 Wang L, Takabe K, Bidlingmaier SM, et al. Sustained correction of bleeding disorder in hemophilia B mice by gene therapy. Proc Natl Acad Sci USA 1999;96:3906-10

30 Strayer DS, Zern MA. Gene delivery to the liver using Simian-Virus 40-derived vectors. Semin Liver Dis 1999;19:71-81.
31 Bilbao G, Feng M, Rancourt C, et al. Adenoviral/retroviral vector chimers: a novel strategy to achieve high-efficiency stable transduction in vivo. FASEB f 1997;11:624-34.

32 Patijn GA, Kay MA. Hepatic gene therapy using adeno-associated virus vectors. Semin Liver Dis 1999;19:61-9.

33 Fraefel C, Jacoby DR, Lage C, et al. Gene transfer into hepatocytes mediated by helper virus-free HSV/AAV hybrid vectors. Mol Med 1997;3:813-25.

34 Farhood H, Gao X, Barsoum J, Huang L. Codelivery to mammalian cells of a transcriptional factor with cis-acting element using cationic liposomes. Anal Biochem 1995;225:89-93. 35 Smith RM, Wu GY. Hepatocyte-directed gene delivery by receptor
mediated endocytosis. Semin Liver Dis 1999;19:83-92. 